\title{
La radioprotection chimique
}

\author{
M. FATOME $(*)$ \\ (Manuscrit reçu le 24 novembre 1980)
}

\begin{abstract}
RÉSUMÉ
Malgré la mise en évidence de composés radioprotecteurs efficaces, les principaux étant des thiophosphates, l'application humaine de la radioprotection chimique reste encore difficile. Les raisons essentielles en sont la perte d'activité à faible dose d'administration et la brève durée d'action de ces composés. Les études tendent actuellement à rechercher des formes d'administration susceptibles de diminuer la dégradation et l'élimination de ces composés. Les premiers essais d'incorporation dans des liposomes sont encourageants. Enfin, la radioprotection fonctionnelle du système nerveux central doit jouer un rôle important dans la protection vis-à-vis de la létalité.
\end{abstract}

\section{SUMMARY}

Human administration of radioprotective compounds - the main ones being thiophosphates - still remains difficult in spite of their effectiveness chiefly because of loss of activity at low doses and short action duration. Present studies are investigating administration forms likely to diminish degradation and elimination of the compounds. The first experiments of incorporation into liposomes are hopeful. On the other hand, functional radioprotection of the central nervous system must play an important part in the protection against lethality.

La radioprotection chimique consiste en l'administration, avant l'irradiation, de substances chimiques dans le but de diminuer les effets nocifs des rayonnements ou, tout au moins, d'en atténuer les conséquences. Son application à l'homme peut s'envisager dans les cas suivants : en milieu industriel pour des missions de sauvetage par exemple, en radiothérapie, en temps de guerre.

Les premières études in vivo remontent en 1945. Vers 1970, environ douze mille composés avaient été testés aux États-Unis. En France, les travaux dans les Armées ont débuté en 1962. Ils sont pratiqués depuis 1968 au Centre de

(*) Centre de recherches du Service de santé des armées, Division de radiobiologie et radioprotection, 1 bis, rue du Lieutenant Raoul-Batany, 92141 Clamart.

RADIOPROTECTION, VOL. 16 - 0033-8451/1981/113/\$ 5.00/ C Bordas-Dunod. 
recherches du Service de santé des armées (C.R.S.S.A.) en collaboration avec la Direction des recherches, études et techniques et avec plusieurs laboratoires universitaires.

Le critère généralement retenu est la prévention de la létalité radioinduite, de sorte que la qualité d'un radioprotecteur est, avant tout, jugée sur sa capacité d'assurer la survie. Aussi, le principal facteur d'évaluation de la radioprotection est-il le facteur de réduction de dose, ou F.R.D., qui est le rapport de la dose d'irradiation létale pour $50 \%$ des individus ayant reçu le protecteur et de celle létale pour $50 \%$ des individus ne l'ayant pas reçu. En règle générale, chez le petit mammifère, sont considérées les DL 50 obtenues au bout de 30 jours après l'irradiation, délai au bout duquel la mort par effet immédiat ne se produira plus.

Le radioprotecteur idéal devrait avoir les caractéristiques suivantes : avoir un F.R.D. au moins égal à 2 , être actif à dose infra-toxique, être dépourvu d'effets pharmacologiques secondaires nocifs aux doses administrées, avoir une durée d'action prolongée sur au moins 6 heures, être actif pour toutes les voies d'administration, être chimiquement stable, avoir une synthèse relativement aisée et facilement reproductible.

Ainsi que nous le verrons, aucun composé actuellement connu ne possède tous ces critères et l'application humaine en reste diffıcile, particulièrement du fait d'une durée d'action souvent brève et surtout d'une faible marge entre la dose efficace et la dose toxique. La plupart des résultats rapportés dans cet article et relatifs à l'action sur la létalité radioinduite et à la protection du système nerveux central sont tirés de travaux conduits dans les laboratoires du C.R.S.S.A.

\section{I. - ACTION SUR LA LÉTALITE RADIOINDUITE}

Les principaux radioprotecteurs connus sont des aminothiols ou des dérivés. Dès 1952, avaient été mises en évidence les propriétés radioprotectrices de plusieurs substances. Les principales étaient la sérotonine ou 5hydroxytryptamine qui est l'un des rares radioprotecteurs non soufrés, la cystéamine ou 2-amino éthanéthiol et l'amino éthyl isothiouronium ou A.E.T. Toutes ces substances présentent, chez la souris, un F.R.D. de 1,6 lorsqu'elles sont administrées par voie intra-péritonéale quelques minutes avant l'irradiation, à dose égale à la moitié de la DL 50 (tableau I).

Malgré de très nombreuses synthèses intéressant des familles chimiques variées, il a fallu attendre plusieurs années pour qu'apparaissent des composés beaucoup plus actifs. Ce sont des thiophosphates synthétisés vers 1965 aux États-Unis au "Walter Reed Institute». Plusieurs présentent un F.R.D. supérieur à 2. Le plus actif est l'acide S-2 (3 aminopropylamino) éthyl phosphorothioïque, plus connu sous le sigle WR 2721, avec un F.R.D. proche de 3 [23] (tableau II).

Le WR 2721 a, depuis, fait l'objet de multiples études. En particulier, son action radioprotectrice a été constatée chez le gros mammifère, le singe entre autres. Bien que moins actif, il se révèle également le meilleur radioprotecteur 
chez l'animal soumis à une irradiation neutronique dont les effets sont beaucoup plus difficiles à prévenir (F.R.D. : 1,6). Une étude de prédiction de son effet protecteur chez l'homme a conclu qu'une dose de $20 \mathrm{mg} / \mathrm{kg}$ serait nécessaire pour obtenir un F.R.D. de 1,5 [22].

TABLEAU 1

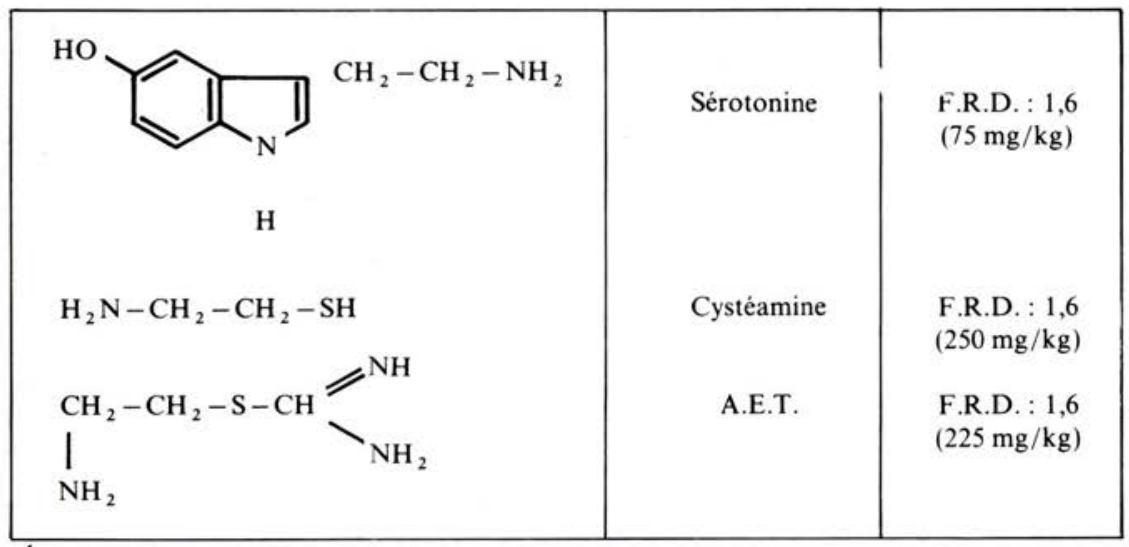

TABLEAU II

\begin{tabular}{|l|c|c|}
\hline & $\begin{array}{c}\text { Dose } \\
(\mathrm{mg} / \mathrm{kg})\end{array}$ & F.R.D. \\
\hline $\mathrm{H}_{2} \mathrm{~N}-\mathrm{CH}_{2}-\mathrm{CH}_{2}-\mathrm{SPO}_{3} \mathrm{H}_{2}$ & 400 & 1,63 \\
$\mathrm{H}_{2} \mathrm{~N}-\left(\mathrm{CH}_{2}\right)_{2}-\mathrm{NH}-\mathrm{CH}_{2}-\mathrm{CH}_{2}-\mathrm{SPO}_{3} \mathrm{H}_{2}$ & 800 & 1,80 \\
$\mathrm{WR} 2721:$ & 500 & 2,70 \\
$\mathrm{H}_{2} \mathrm{~N}-\left(\mathrm{CH}_{2}\right)_{3}-\mathrm{NH}-\mathrm{CH}_{2}-\mathrm{CH}_{2}-\mathrm{SPO}_{3} \mathrm{H}_{2}$ & 400 & 2,05 \\
$\mathrm{H}_{2} \mathrm{~N}-\left(\mathrm{CH}_{2}\right)_{4}-\mathrm{NH}-\mathrm{CH}_{2}-\mathrm{CH}_{2}-\mathrm{SPO}_{3} \mathrm{H}_{2}$ & 300 & 1,92 \\
$\mathrm{H}_{2} \mathrm{~N}-\left(\mathrm{CH}_{2}\right)_{5}-\mathrm{NH}-\mathrm{CH}_{2}-\mathrm{CH}_{2}-\mathrm{SPO}_{3} \mathrm{H}_{2}$ & 300 & 1,90 \\
$\mathrm{H}_{2} \mathrm{~N}-\left(\mathrm{CH}_{2}\right)_{6}-\mathrm{NH}-\mathrm{CH}_{2}-\mathrm{CH}_{2}-\mathrm{SPO}_{3} \mathrm{H}_{2}$ & 300 \\
\hline
\end{tabular}

Il est encore actuellement difficile d'expliquer l'activité particulière de ce thiophosphate. Il est probable que la molécule d'acide phosphorique et que la partie de la molécule greffée sur l'azote de la cystéamine favorisent la pénétration intra cellulaire et la libération plus intime de groupements $\mathrm{SH}$ libres.

Parmi les autres familles testées, celle des thiazolidines mérite une place à part. Elles possèdent dans leur cycle la structure de la cystéamine. Certes, leur activité est nettement inférieure à celle des thiophosphates, les plus actives ayant un F.R.D. de l'ordre de 1,5 comparable à celui de la cystéamine [20]. Mais elles ont souvent une durée d'action plus longue égale à quelques heures (tableau III). 
TABLEAU III

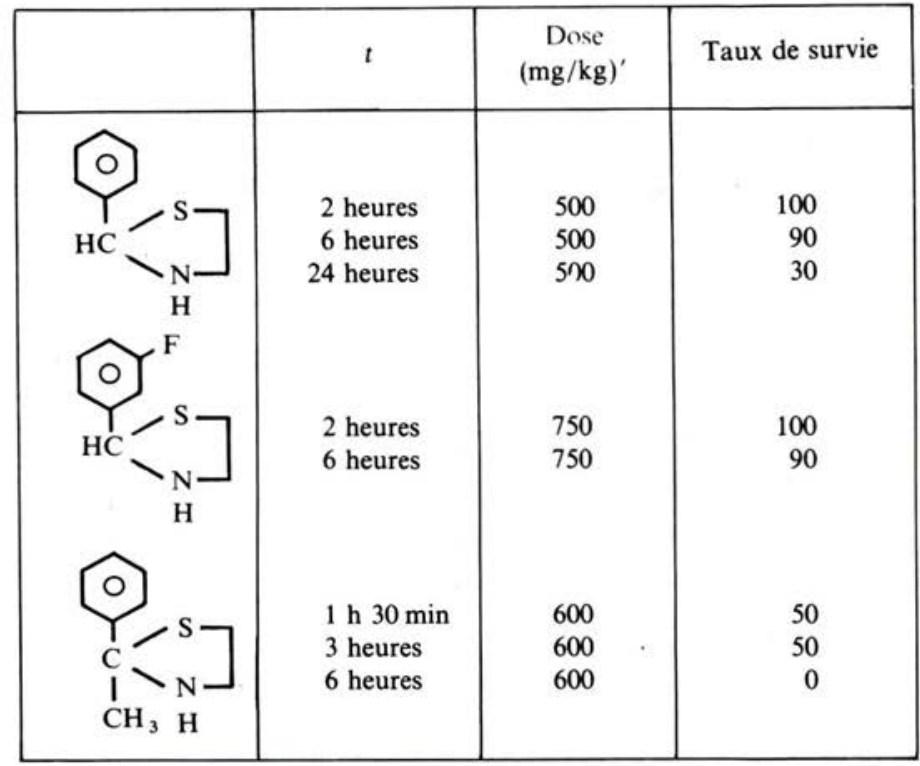

En greffant un gros atome comme le brome, elles peuvent avoir une action différée. Par ailleurs, plusieurs sont actives par voie orale chez la souris. Elles semblent malheureusement mal supportées sur le plan digestif, chez le gros mammifère.

Il est difficile de préciser si leur action est liée au caractère propre de la thiazolidine ou si elle s'exerce par l'intermédiaire de cystéamine libérée. Les travaux menés dans ce sens par Vos en culture cellulaire ont montré qu'il n'y a pas de relation entre le pouvoir protecteur et la vitesse d'hydrolyse (résultats en cours de publication). Par contre, des travaux récents conduits par СнAPAT [4] ont mis en évidence une coupure de la molécule de phényl-2 thiazolidine marquée après administration à la souris.

Parmi d'autres familles chimiques ayant fourni des dérivés actifs, on peut, entre autres, citer les thiazolines, les thiosulfates, les adamantanes mais actuellement elles paraissent n'apporter rien de plus que les précédentes.

Il existe donc, actuellement, des dérivés assez actifs avec un F.R.D. supérieur à 2. Toutefois, l'application pratique en reste limitée en raison de la chute de leur activité avec la diminution de la dose administrée, ou avec l'allongement du délai compris entre l'administration et l'irradiation. Sur un plan militaire, leur manque d'efficacité par voie orale est un inconvénient supplémentaire. C'est pourquoi, depuis quelques années, les recherches ont été plus orientées vers la résolution de ces problèmes que vers la découverte de nouvelles familles chimiques. 
a) ReCHERCHE DUUNE DIMINUTION DE TOXICITÉ

Il existe le plus souvent une faible marge entre la dose radioprotectrice et la dose toxique, le fait de diminuer la dose administrée faisant chuter rapidement l'activité radioprotectrice.

Cet inconvénient est moins net avec les thiophosphates et particulièrement avec le WR 2721 qui possède encore une action à dose infratoxique.

Pour tenter de pallier cet inconvénient, des molécules ou des groupements chimiques ont été greffés sur des molécules actives, et des associations ont été pratiquées.

Ainsi, il y a quelques années, ont été testées des condensations de sucre et de cystéamine ou de thiazolidine synthétisées par $\mathrm{IMBACH}$. La toxicité était alors très réduite, mais le pouvoir radioprotecteur diminuait de façon aussi spectaculaire.

En thérapeutique, il a été montré que certaines molécules acétyléniques sont plus actives et moins toxiques que leurs homologues saturés. C'est pourquoi ont été synthétisés plusieurs dérivés insaturés de cystéamine et de thiazolidine (Miginiac). Le but recherché n'a été atteint que de façon inconstante [14].

Le fait de greffer un radical méthyle sur le carbone en position 1 par rapport au soufre de la cystéamine (СHAPAT) en diminue la toxicité et en augmente l'efficacité à dose infra-toxique (tableau IV).

TABLEAU IV

\begin{tabular}{|c|c|c|c|}
\hline \multicolumn{2}{|c|}{$\begin{array}{c}\text { Cystéamine } \\
\mathrm{H}_{2} \mathrm{~N}-\mathrm{CH}_{2}-\mathrm{CH}_{2}-\mathrm{SH}, \mathrm{ClH} \\
\text { DL } 50: 450 \mathrm{mg} / \mathrm{kg} \\
: 3,96 \mathrm{mmol} / \mathrm{kg}\end{array}$} & \multicolumn{2}{|c|}{$\begin{array}{c}\text { Méthyl-1 amino-2 éthanethiol } \\
\mathrm{H}_{2} \mathrm{~N}-\mathrm{CH}_{2}-\mathrm{CH}-\mathrm{SH}, \mathrm{ClH} \\
\mathrm{CH}_{3} \\
\text { DL } 50: 800 \mathrm{mg} / \mathrm{kg} \\
: 6,27 \mathrm{mmol} / \mathrm{kg}\end{array}$} \\
\hline $\begin{array}{c}\text { Dose } \\
(\mathrm{mg} / \mathrm{kg})\end{array}$ & $\begin{array}{l}\text { Taux de survie } / 30 \mathrm{j} \\
\text { (irradiation } \mathrm{DL} 100 / 30 \mathrm{j} \text { ) }\end{array}$ & $\begin{array}{c}\text { Dose } \\
(\mathrm{mg} / \mathrm{kg})\end{array}$ & $\begin{array}{c}\text { Taux de survie } / 30 \mathrm{j} \\
\text { (irradiation } \mathrm{DL} 100 / 30 \mathrm{j} \text { ) }\end{array}$ \\
\hline $\begin{array}{r}225 \\
200 \\
175 \\
150 \\
125 \\
100 \\
75\end{array}$ & $\begin{array}{l}95 \% \\
90 \% \\
70 \% \\
55 \% \\
45 \% \\
40 \% \\
0 \%\end{array}$ & $\begin{array}{l}400 \\
200 \\
100 \\
50 \\
25 \\
12,5\end{array}$ & $\begin{array}{l}95 \% \\
95 \% \\
80 \% \\
60 \% \\
5 \% \\
0 \%\end{array}$ \\
\hline \multicolumn{2}{|c|}{$\begin{array}{c}\text { Dose donnant } 50 \% \text { de survie } / 30 \mathrm{j} \\
140 \mathrm{mg} / \mathrm{kg} \\
\simeq 1 / 3 \mathrm{DL} 50\end{array}$} & \multicolumn{2}{|c|}{$\begin{array}{c}\text { Dose donnant } 50 \% \text { de survie } / 30 \mathrm{j} \\
45 \mathrm{mg} / \mathrm{kg} \\
\simeq 1 / 17 \mathrm{DL} 50\end{array}$} \\
\hline
\end{tabular}

VOL. $16-\mathrm{N}^{\circ} 2$ 
Enfin, le Diazepam qui présente par lui-même, un léger effet radioprotecteur s'est révélé, lorsqu'il était administré environ 1 heure avant la cystéamine, en diminuer la toxicité et en augmenter l'effet protecteur, le F.R.D. passant de 1,6 à $1,9[6]$ (tableau V).

TABLEAU $V$

INFLUENCE DU DIAZEPAM SUR L'ACTION RADIOPROTECTRICE DE LA CYSTÉAMINE

\begin{tabular}{|c|c|c|}
\hline \multirow[b]{2}{*}{$\begin{array}{l}\text { Dose d'irradiation } \\
\text { (R) }\end{array}$} & \multicolumn{2}{|c|}{ Taux de survie $/ 30 \mathrm{j}$} \\
\hline & $\begin{array}{c}\text { Cystéamine IP } \\
300 \mathrm{mg} / \mathrm{kg}\end{array}$ & $\begin{array}{c}\text { Diazepam IP } 15 \mathrm{mg} / \mathrm{kg} \\
\text { + cystéamine IP } 350 \mathrm{mg} / \mathrm{kg} \\
1 \mathrm{~h} 15 \mathrm{~min} \text { après }\end{array}$ \\
\hline $\begin{array}{l}1050 \\
1200 \\
1350 \\
1500 \\
1650 \\
1800\end{array}$ & $\begin{array}{r}100 \% \\
90 \% \\
55 \% \\
20 \% \\
0 \% \\
0 \%\end{array}$ & $\begin{array}{r}100 \% \\
100 \% \\
100 \% \\
80 \% \\
40 \% \\
0 \%\end{array}$ \\
\hline
\end{tabular}

b) Tentative d'allongement de la durée d'action

La plupart des radioprotecteurs ont une durée d'action inférieure à 1 heure. Jusqu'en 1967, on ne connaissait aucune substance à action prolongée.

En 1969, il avait été étudié une condensation d'acide glyoxylique et de cystéamine. Testée sous le nom de C 511, cette substance injectée $15 \mathrm{~min}$ avant irradiation, était aussi efficace que la cystéamine [11]. Mais sa durée d'action était nettement supérieure assurant une protection encore significative pour un délai de 24 heures [9]. Soulignons qu'un tel effet n'était observé que s'il s'agissait bien d'une condensation des deux molécules et non d'un mélange à parties égales, l'acide glyoxylique devant favoriser la pénétration intra cellulaire et assurer une libération progressive de la cystéamine.

On a pensé que la courte durée d'action des composés vraisemblablement liée à une inactivation et à une élimination rapides pouvait avoir pour origine leur faible poids moléculaire. L'idée de tester des polymères a donc été avancée. L'intérêt en avait été lancé par RINGSDORFF qui avait montré l'action prolongée pendant 8 jours d'un copolymère de $\mathrm{N}$-acryloyl-3 méthylthiazolidine, d'acide acryloïque et de vinylpyrrolidone avec un F.R.D. de 1,4, résultat remarquable en soi [18].

Certains copolymères préparés par BARNES et contenant du vinylpyrrolidone ont présenté une activité prolongée sur 24 ou 48 heures. Celle-ci était toutefois beaucoup plus faible que celle constatée par RINGSDORFF avec le polymère précédent. La solubilité dans l'eau paraît essentielle et le poids moléculaire ne doit pas être trop élevé pour que les groupements actifs ne soient pas masqués par la configuration complexe de la chaîne polymérisée [2]. 
Toutefois, l'intérêt pratique de tels copolymères paraît très restreint si l'on considère les risques retardés liés à leur injection, en particulier, ceux inhérents à leur caractère antigénique et à la toxicité de la polyvinylpyrrolidone indispensable pour favoriser l'hydrosolubilité.

Aussi, plus intéressants paraissent être les résultats obtenus avec un polyflavane extrait de Poterium Spinosum, préparé par les laboratoires FABRE. Il s'agit d'un extrait lyophilisé, de faible poids moléculaire compris entre 2000 et 3000 . Son intérêt ne vient pas tant de la valeur de son F.R.D. (1,3) que de l'effet prolongé sur 24 heures pour une dose optimale d'administration faible par rapport à la dose toxique (1/8 de la DL 50), l'effet n'étant pas nul, même pour une administration faite au 1/20 de la DL 50 [7] (tableau VI).

\section{TABLEAU VI}

INFLUENCE DE LA DOSE DE POLYFLAVANE ET DE LINTERVALLE DE TEMPS SÉPARANT LINJECTION DE L'IRRADIATION SUR LE TAUX DE SURVIE AU $30^{\circ}$ JOUR.

\begin{tabular}{|c|r|r|r|r|r|}
\hline $\begin{array}{c}\text { Délai entre l'injection } \\
\text { et l'irradiation } \\
\text { (DL 100/30 j) }\end{array}$ & \multicolumn{5}{|c|}{$\begin{array}{c}\text { Taux de survie au 30 jour } \\
\text { aux différentes doses injectées } \\
\text { (mg/kg)-IP }\end{array}$} \\
\cline { 2 - 6 } (h) & 75 & 60 & 40 & 20 & 0 \\
\hline 1 & $16 / 20$ & $10 / 10$ & $8 / 10$ & $1 / 10$ & $0 / 10$ \\
2 & $17 / 20$ & $8 / 10$ & $9 / 10$ & $5 / 10$ & $0 / 10$ \\
24 & $34 / 40$ & $12 / 20$ & $11 / 20$ & $7 / 18$ & $0 / 10$ \\
\hline
\end{tabular}

Actuellement, on ne peut pas encore affirmer si le mode d'action des polymères est lié aux propriétés que leur confère leur nature polymérisée ou s'il vient de la libération progressive de monomères actifs.

\section{c) EFFICACITÉ PAR VOIE ORALE}

Les résultats sont le plus souvent décevants. Même le thiophosphate WR 2721 est peu actif par cette voie. Au total, si l'on considère les caractéristiques du radioprotecteur idéal force est de constater qu'aucun composé ne les possède actuellement toutes. Quelles perspectives peuvent être envisagées?

\section{d) Perspectives}

1. La voie de la synthèse chimique, en particulier la greffe de molécules susceptibles de se comporter comme des prodrogues, paraît donner des résultats limités. On peut toutefois envisager les perspectives suivantes :

- La mise en évidence de l'activité radioprotectrice présentée par un trisulfure à fonction sulfinate et sans fonction amine paraît montrer l'intérêt d'approfondir l'étude de tels dérivés soufrés mais non azotés. 
Le remplacement d'un atome de carbone (tel celui en position 2 des thiazolidines) par des substituants susceptibles d'augmenter l'hydrosolubilité et de favoriser le franchissement des membranes cellulaires pourrait apporter des résultats intéressants. De plus, en modifiant la structure électronique et le comportement chimique des molécules, ces substituants leur confèrent des propriétés nouvelles et spécifiques par rapport à celles de leurs dérivés isologues carbonés.

- Enfin, par analogie avec la cyclophosphamide qui, après administration par voie orale, libère le principe actif après métabolisation hépatique, on peut espérer obtenir des molécules qui, données par cette voie, seraient susceptibles de libérer de la même façon les composés les plus actifs.

2. En fait, les problèmes posés ont peut être une solution faisant plus appel à l'étude des formes d'administration qu'à la chimie de synthèse. Il semble, en effet, que la majeure partie des substances administrées telles quelles soit perdue par dégradation, par élimination rapide, et par fixation sur un site peu intéressant pour la radioprotection. Il en résulte une très faible pénétration au niveau du site actif. Ceci rend compte de la très faible efficacité et de la courte durée d'action. Pour pallier ces inconvénients, il est nécessaire d'augmenter la quantité administrée qui se trouve alors dans la zone toxique.

L'idée qui vient alors à l'esprit est l'utilisation de véhicules capables de protéger les molécules des phénomènes de dégradation et d'élimination pendant leur transport vers le site actif et de favoriser leur pénétration à ce niveau. Ceci doit entraîner une diminution de la quantité administrée et des manifestations toxiques. Ce mode d'administration est envisagé dans d'autres domaines que celui de la radioprotection chimique, telles l'enzymologie ou la chimiothérapie anti cancéreuse. Actuellement, le moyen le plus étudié est l'encapsulation au sein de liposomes qui sont des vésicules lipidiques préparées artificiellement [17]. Leur nature, leur taille et leur composition sont importantes en ce qui concerne la quantité de substance incorporée, sa vitesse de libération, et la nature des sites de fixation, les principaux étant le plus souvent le foie et la rate. Les premiers essais d'encapsulation de substance radioprotectrice au sein de ces vésicules se révèlent encourageants pour la poursuite de l'étude. Par la suite, d'autres moyens pourront être envisagés.

De ces perspectives, on peut conclure que les espoirs se fondent plus sur l'étude des formes d'administration que sur la chimie de synthèse.

\section{II. - AUTRES APPLICATIONS \\ DE LA RADIOPROTECTION}

Si la majeure partie des travaux concerne la létalité induite par les effets immédiats de l'irradiation, il n'en reste pas moins que d'autres aspects sont importants à considérer. 


\section{Prévention des efFets tardifs}

Les effets tardifs ont été relativement peu étudiés, les principaux travaux ont été effectués par MaISIN [12-13]. Il a, en particulier, montré, chez la souris soumise à une irradiation unique ou fractionnée, l'influence bénéfique d'un mélange de radioprotecteurs sur la durée de vie, la cause essentielle étant la réduction de l'incidence de certains cancers et, en particulier, des lymphomes thymiques. Cette observation est importante car, contrairement à ce que l'on peut a priori redouter, il ne semble pas que l'administration de radioprotecteurs entraîne, par diminution du nombre de cellules tuées, une augmentation de l'incidence des tumeurs par élévation du nombre de cellules présentant des lésions sublétales.

\section{Protection des tumeurs}

L'utilisation de radioprotecteurs en radiothérapie ne peut se concevoir que dans la mesure où la protection ne s'exerce pas sur les cellules tumorales. Plusieurs études pratiquées avec des thiophosphates et surtout avec le WR 2721 ont mis en évidence une faible protection paraissant inversement liée à l'importance de la vascularisation tumorale et à celle de l'oxygénation cellulaire $[15,21,24,25]$. D'autres études ont montré l'intérêt de l'association d'un radioprotecteur et d'un radiosensibilisant en l'occurence le WR 2721 et le misonidazole qui n'auraient pas d'influence sur leurs activités réciproques en termes de protection de tissu sain et de sensibilisation de tumeurs $[19,26]$. Un projet d'utilisation du WR 2721 en radiothérapie est en cours aux EtatsUnis $[16,22]$.

\section{Prévention des vomissements radioinduits}

La prévention des vomissements radioinduits intéresse aussi bien le domaine militaire du temps de guerre que celui de la radiothérapie ou celui de l'accident. Elle est envisagée, ici, bien qu'elle ne rentre pas dans la définition stricte de la radioprotection chimique telle qu'elle a été donnée en introduction, l'administration des composés pouvant être faite après l'irradiation. Les antiémétiques connus ne sont généralement pas parfaitement efficaces et ne sont pas dépourvus d'effets secondaires d'origine centrale. Un des meilleurs connus est le Domperidome ${ }^{R}$ des laboratoires JANSSEN [3]. Depuis quelques années, des études systématiques sont entreprises dans ce domaine par différents laboratoires. Il faut toutefois souligner que de tels travaux sont compliqués par l'imparfaite connaissance du mécanisme de déclenchement du vomissement radioinduit et par la difficulté du choix d'un modèle expérimental approprié.

\section{Protection du système nerveux Central}

La radiosensibilité fonctionnelle du système nerveux central de l'adulte a été mise en évidence et confırmée depuis une vingtaine d'années. Elle se manifeste même à doses infralétales par des signes d'irritabilité et de souffrance cérébrale et 
par une hyperexcitabilité de certaines structures. Des travaux conduits au cours de ces dernières années ont montré que, chez le lapin adulte soumis à une irradiation globale $(6,5$ Gy ou 650 rads), l'injection préalable de sérotonine,de cystéamine, d'A.E.T. ou de thiophosphate WR 2721 prévient de façon importante les principales altérations électroencéphalographiques radioinduites $[5,8]$. Le caractère inconstant et modéré des perturbations constatées, confirmé par le traitement énergétique du signal EEG, est comparable à celui noté après une irradiation globale, sans protection, comprise entre 1,5 et 2,5 Gy (150 et 250 rads). L'absence ou la diminution importante de l'hyperexcitabilité radioinduite de certaines structures cérébrales, comme la formation réticulée mésencéphalique doit jouer un rôle important. Toutefois, le mécanisme intime de cette protection reste inconnu et soulève de nombreuses questions quant à l'action des rayonnements ionisants sur le système nerveux central (parts respectives de l'action centrale et de l'action périphérique - altérations du métabolisme du neurone - troubles de la perméabilité membranaire modifications du taux des neuromédiateurs - action des produits de dégradation...). Toutes ces incertitudes ouvrent le champ à de nombreuses investigations.

Quel que soit le mécanisme exact de cette protection, celle-ci doit certainement jouer un rôle dans la protection générale de l'individu. Le mécanisme d'action des radioprotecteurs reste, en effet, encore mal connu. Les principales théories émises font appel à l'échelon moléculaire et cellulaire [1-10] (diminution de tension d'oxygène - capture des radicaux libres - formation de ponts disulfure entre les protéines soufrées et les molécules radioprotectrices soufrées - protection des acides nucléiques - perturbations passagères de métabolisme cellulaire). Toutefois, si l'on s'intéresse à la protection du mammifère, on peut penser qu'il existe également des mécanismes plus globaux.

En particulier, si l'on admet que les altérations fonctionnelles centrales peuvent être à l'origine de perturbations fonctionnelles périphériques et si l'on considère le rôle joué par le système nerveux central dans les grandes régulations de l'organisme, on peut avancer l'idée que la protection centrale doit être un des éléments à l'origine de la survie de l'individu irradié à dose létale et, de ce fait, être un des mécanismes d'action des radioprotecteurs.

\section{CONCLUSION}

Malgré la découverte de composés radioprotecteurs efficaces, l'application humaine de la radiopretection chimique reste encore difficile. Les raisons essentielles en sont la perte d'activité à faible dose d'administration et la brève durée d'action de ces composés. Comme dans d'autres domaines thérapeutiques et prophylactiques, les études tendent à se tourner vers la recherche de formes d'administrations susceptibles de diminuer la dégradation et l'élimination de ces composés et d'entraîner une action par voie orale. Le thiophosphate WR 2721 qui possède une action déjà importante à dose infratoxique et paraît retenu aux Etats-Unis pour une application humaine $[16,22]$ pourrait alors se révéler 
particulièrement intéressant. Enfin, sur un plan fondamental, la radioprotection fonctionnelle du système nerveux central doit jouer un rôle important dans la protection vis-à-vis de la létalité.

\section{BIBLIOGRAPHIE}

[1] BACQ Z. M., Goutier R. Mechanism of action of sulfur containing radioprotectors. In Recovery and repair mechanisms in radiobiology, Brookhaven Symposium on Biology, 1967, 20, 241-260.

[2] Barnes J. H., Fatome M., Esslemont G. F., A ndrieu L. Prolonged radioprotective action of water-soluble polymers with labile sulphur containing side chains, Eur.J. Med.Chem., 1977, 12, 467-470.

[3] Bernier J., Huys J. The effect of Domperidone on the symptomatic treatment of radiotherapy induced nausea and vomiting. Postgrad.Med. J. Suppl., 1979, 55, n 1, 52-53.

[4] Снарат J. P. Étude métabolique chez la souris de dérivés de la phényl-2 thiazolidine. Relation entre distribution, métabolisation et activité radioprotectrice. Communication présentée au GESA IX, Campo Dell'Oro, Corse, 15-18 mai 1979.

[5] FATOME M., COURT L. Contribution à l'étude du rôle radioprotecteur de la sérotonine sur l'activité électrique cérébrale spontanée et évoquée du lapin adulte soumis à une irradiation globale létale. Rapport CEA-R-4483, 1973, 105 p.

[6] Fatome M., Gaudefroy A., Laval J. D., Andrieu L., Sentenac-Roumanou M. Le Diazepam : action radioprotectrice et influence sur la toxicité et le pouvoir radioprotecteur de la cystéamine. Eur. J. Med. Chem., 1977, 12, 93-95.

[7] Fatome M., Sentenac-Roumanou M., Laval J. D., Andrieu L., Foulhoux P. Action radioprotectrice d'un polyflavane d'origine végétale chez la souris irradiée à dose létale. Eur. J. Med., 1978, 13, 241-244.

[8] Fatome M. Contribution à l'étude du rôle de la radioprotection du système nerveux central dans le mécanisme d'action des substances radioprotectrices soufrées. Thèse de Doctorat d'État ès Sciences, Université Paris-VI, 16 juin 1980, $235 \mathrm{p}$.

[9] Frossard H., Fatome M., Cadot M., Bonnardot C., Sauvant D. Action radioprotectrice du C 511 injecté chez la souris 24 h avant une irradiation à dose létale. C.R. Acad. Sci., Paris, 1972, 275, série D, 1923-1925.

[10] Foye W. O. Mechanisms of radiation protection by the aminothiols. Int. J. Sulfur Chem., 1975, 8, 161-171.

[11] Jezequel. E. J., Frossard M., Fatome M., Perles R., Poutrain P. Activité radioprotectrice des produits de condensation de l'acide glyoxylique et des ses esters avec quelques aminothiols. C.R. Acad. Sci., Paris, 1971, 272, série D, 2826-2827.

[12] Maisin J. R., Gerber G. B., Mattelin G., Lambiet-Colier M. Chemical protection against life shortening and causes of death after a single or fractionated whole-body exposure of mice to ionizing radiation. In: Late biological effects of ionizing radiation, Vienna, 13-17 mars 1978. IAEA: Vienna, 1978, vol. II, 483-494.

[13] Maisin J. R., Gerber G. B., Lambiet Collier M. Chemical protection against long-term effects of whole body exposure of mice to ionizing radiation. 3 . The effects of fractionated exposure to C 57 B I mice. Radiat. Res., 1980, 82, 487-497.

[14] Mesnard D., Miginiac L., Fatome M., Laval J. D., Sentenac-Roumanou H., Lion C. Synthèse et étude radio-pharmacologique de composés insaturés dérivés de la cystéamine. Eur. J. Med. Chem., 1980, 15, 247-252.

vOL. $16-\mathrm{N}^{\circ} 2$ 
[15] Phillips T. L., Kane L., Utley J. F. Radioprotection of tumour and normal tissues by thiophosphate compounds. Cancer, 1973, 32, 528-535.

[16] Phillips T. L. Chemical modification of radiation effects. Cancer, 1977, 39, 987-999.

[17] Puiseux F., Luong T. T., Moufti A. Les liposomes, véhicules possibles de principes actifs. Pharm. Acta Helr.. 1977, 52, 305-318.

[18] Ringsdorf M., Heisler A. G., Muller F. H., Graul E. H., Ruther W. In : Biological aspects of radiation protection, Kyoto, octobre 1969 (T. SugahaRA, Ed.) Tokyo : Igaku Shoin, 1971, 138.

[19] Sodicoff M., Longer A. D., Pratt N. E., Sinesi M., Trepper. Chemoradioprotection of the rat parotid gland by combined use of WR 2721 and R.O.-07-058 L. Radiat. Res., 1979, 80, 348354.

[20] Terol A., Fernandez J. P., Robbe Y., Chapat J. P., Granger R., Fatome M., Andrieu L., Sentenac-Roumanou $\mathrm{H}$. Recherche d'agents radioprotecteurs dérivés de la phényl-2 thiazolidine. Eur. J. Med. Chem., 1978, 131, 149-151.

[21] Utley J. F., Marlowe C., Waddel W. J. Distribution of 35S WR 2721 in normal and malignant tissues in the mouse. Radiat. Res., 1976, 68, 284-291.

[22] WASHBURN J. M. Prediction of the effective radioprotective dose of WR 2721 in human through an intraspecies tissue distribution study. Radiat. Res., 1976, 66, 100-105.

[23] Yuhas J. M., Storer J. B. Chemoprotection against three modes of radiation death in the mouse. Int. J. Radiat. Biol., 1969, 15, 233-237.

[24] Yuhas J. M., Storer J. B. Differential chemoprotection of normal and malignant tissues. $J$. Natl. Cancer Inst.. 1969, 42, 331-335.

[25] Yuhas J. M. Biological factors affecting the radioprotective efficiency of S-2 (3 amino propylamino) ethyl phosphorothioï acid (WR 2721), LD 50/30 doses. Radiat. Res., 1970, 44, 621-628.

[26] Yuhas J. M., Yurconic M., Kligerman M. M., West G., Peterson D. F. Combined use of radioprotective and radiosensitizing drugs in experimental radiotherapy. Radiat. Res., 1978, 70, 433-443. 